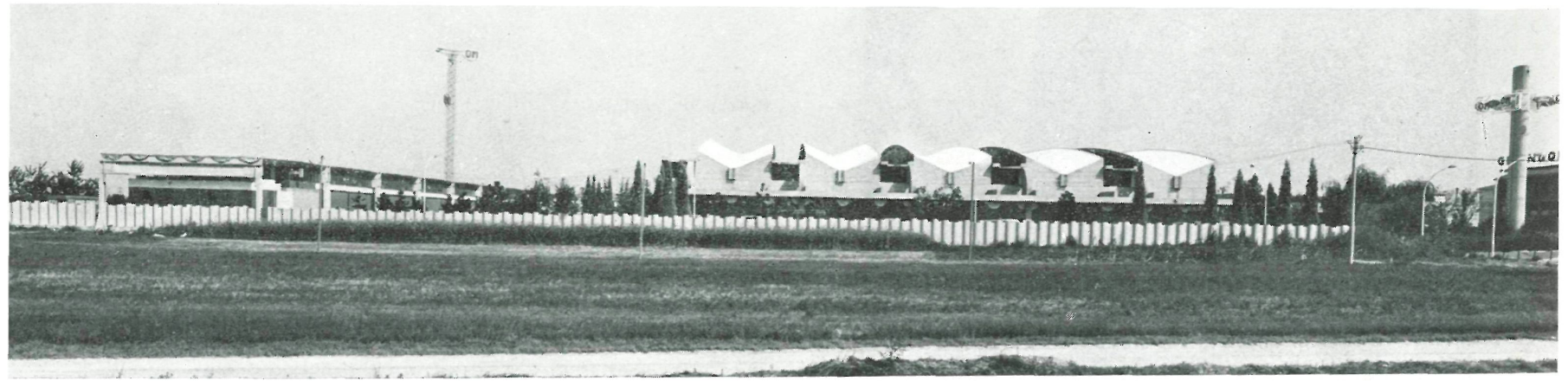

La Sociedad "A. Gandolfi», delegada para las provincias de Bologna, Ferrara y Rovigo de las importantes firmas de automóviles y vehículos industriales OM, Simca, Autobianchi, Ford, ha levantado su nueva sede, y al mismo tiempo el Centro de su organización comercial e industrial, en las afueras de la ciudad de Bologna, próxima a uno de los nudos de la autopista, sobre un emplazamiento que dispone de amplios espacios para aparcamiento, y dotándola de una perfecta organización y de una serie de instalaciones confortables (bar, restaurante, barra para comidas calientes, etc.).

La solución estructural adoptada, la selección de los materiales empleados, la composición arquitectónica de las edificaciones y la integración en el conjunto de vastas zonas verdes, son las características dominantes del complejo.

Al proyectar este Centro fueron estudiados con detenimiento los siguientes aspectos: relación cliente-administración, desechando el antiguo concepto de barrera, de separación, que existiría entre el cliente y la zona de trabajo interior; y fieles al nuevo criterio, tanto las zonas de trabajo creadas como las de descanso, de exposición, de entrega de vehículos producen una sensación de contacto directo e íntimo con el cliente, estando las oficinas localizadas de modo que le ofrecen a aquél un cómodo acceso, sin prescindir, por ello, de las conexiones y relaciones precisas con el jefe de taller, con los recepcionistas, con los encargados del control de los trabajos e, incluso, con la sección de exposición de coches.

En el circuito comercial establecido se ha instalado una zona de descanso, con bar-restaurante y sala de espera, que hace más confortable el ambiente general interior; los caminos, recorridos y espacios destinados al cliente están bien definidos, y en ellos se disfruta de gran libertad de movimientos y de observación.

\title{
centro de servicio y asistencia para vehículos-automóviles ITALIA
}

\author{
G. GRESLERI, Dr. arquitecto
}

sinopsis

$132-54$
Podemos distinguir en este Centro las siguientes unidades: estación de servicio completa; edificio de oficinas $-y$ en contacto con éstas, el taller en las que se realizan los "trabajos pesados» - carrocerías, radiadores, lavado y engrase, etc.-; la edificación que contiene los servicios generales y particulares; etc.

Al proyectar este conjunto fueron estudiados con detenimiento los siguientes aspectos: relación cliente-administración; el trabajo como factor diversos núcleos de una perfecta rocionalidad en cuanto a su funcionamiento una expresiva personalidad.
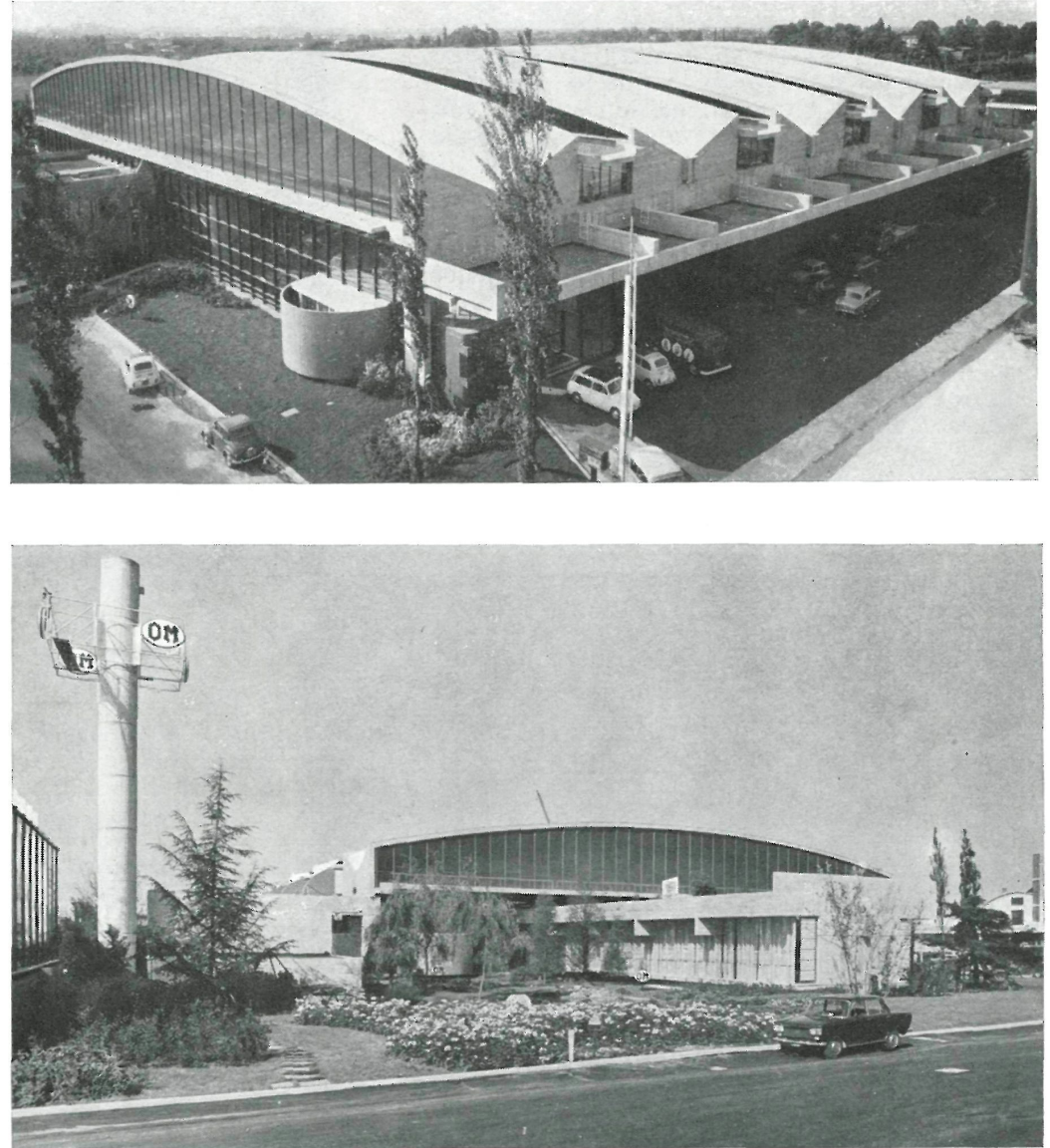


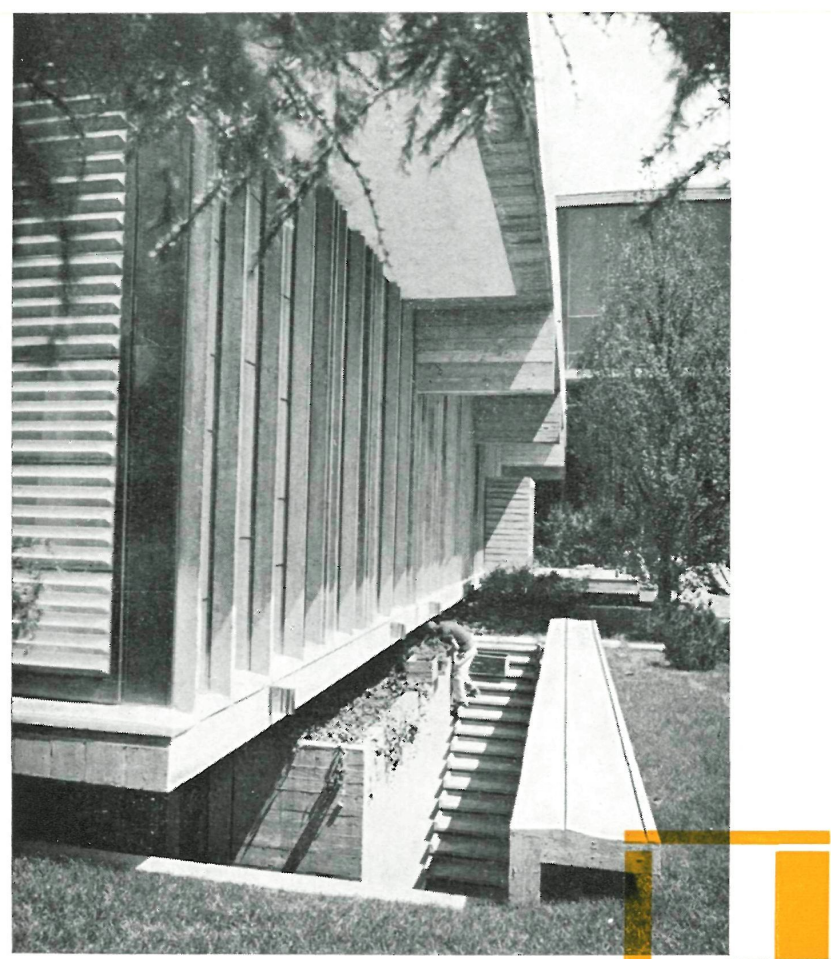

Cuerpo de oficinas

núcleos; no existen ni espacios más bellos, ni más lujosos, ni más pobres, sino que el organismo fue concebido como un todo homogéneo diversamente articulado. La calificación de los espacios es una consecuencia de la diferenciación de las funciones que en los mismos se desarrollan, y así, tanto las zonas en las que se efectúan los trabajos, como los ambientes con los que ha de estar en contacto el cliente, como los almacenes y diferentes servicios, han sido tratados con un mismo nivel cualitativo.

Aunque los materiales empleados en las diversas edificaciones son los usuales -hormigón "visto», acero, vidrio, etc.-, la composición arquitectónica da la impresión de una gran unidad expresiva y formal. y de que las diversas actividades humanas que en ellas se desarrollan (operaciones manuales, de contabilidad, etc.) han sido elevadas todas a un nivel de alta dignidad humana.

Oficinas: interior

Otro aspecto se ha tenido en cuenta: el del trabajo como factor educativo y de superación; todo puesto de trabajo fue concebido como un espacio en el que se desarrolla una actividad humana, habiendo sido estudiados previamente las componentes físicas y sicológicas que lo caracterizan, a fin de conferirle la más alta dignidad y categoría que se le pueda imprimir; siendo óptimas las condiciones de aire, luz, colorido, expresividad de los materiales, acondicionamiento térmico, seguridad, orden, dignidad de los servicios, etc.

Se ha considerado un tercer factor determinante: el lograr una homogeneidad de cualidades en la calificación de los diversos
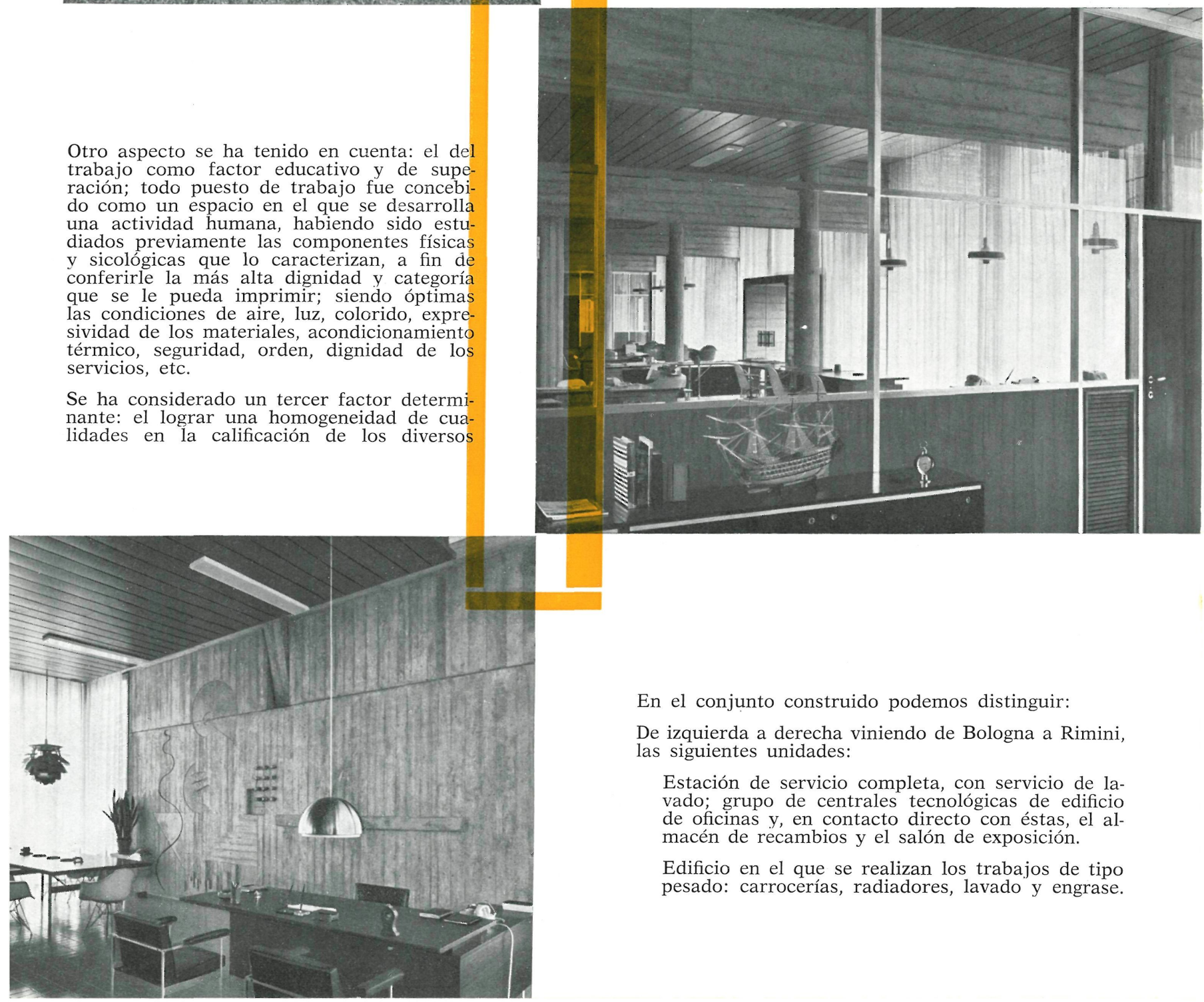

En el conjunto construido podemos distinguir:

De izquierda a derecha viniendo de Bologna a Rimini, las siguientes unidades:

Estación de servicio completa, con servicio de lavado; grupo de centrales tecnológicas de edificio de oficinas y, en contacto directo con éstas, el almacén de recambios y el salón de exposición.

Edificio en el que se realizan los trabajos de tipo pesado: carrocerías, radiadores, lavado y engrase. 
planta general A. Estaciones de servicio.-B. Central tecnologica y exposicion. - . ción y oficina.-E. Trabajos costosos. - F. Śrvicios generales. G. Guarda.

1. Vitrina exposición modelos nuevos. -2 . Central térmica. -3 . Cen tral de acondicionamiento. -4 . Ven de piezas de recambio - 6. Estenque reserva de agua contra incendios.-7. Aparcamiento--8. Espacio de circulación y maniobra autovehiculos.-9. Control acceso oficinas.-10. Recepción y consigna.11. Acceso a la sala de la exposicion. 12 . Exposición de modelos na de trabajo - 14. control_-15. Foso-16. Pasillo de acceso a fosos.-17. Máquinas.18. Piezas de recambio.-19. Mostrador.-20. Cája, contabilidad y administración.-21. Aseo-vestuario (empleados).-22. Oficinas (comercial legal ).-23. Director-24. Escalera a la cabina del jefe de of ba de motoro a la sala de prueacceso a servicios 26 . Rempa de medor de operarios - 27. Restaurante.-28. Cocina.-29. Bar y mesa caliente.-30. Rampa a la escalera de los apartamentos.-31. Aseos.-32. Salida de vestuarios generales. - 33. Relojes de control. 34. Escalera a los servicios de restaurante. -35. Lavado de coches. miento -38 . Acces
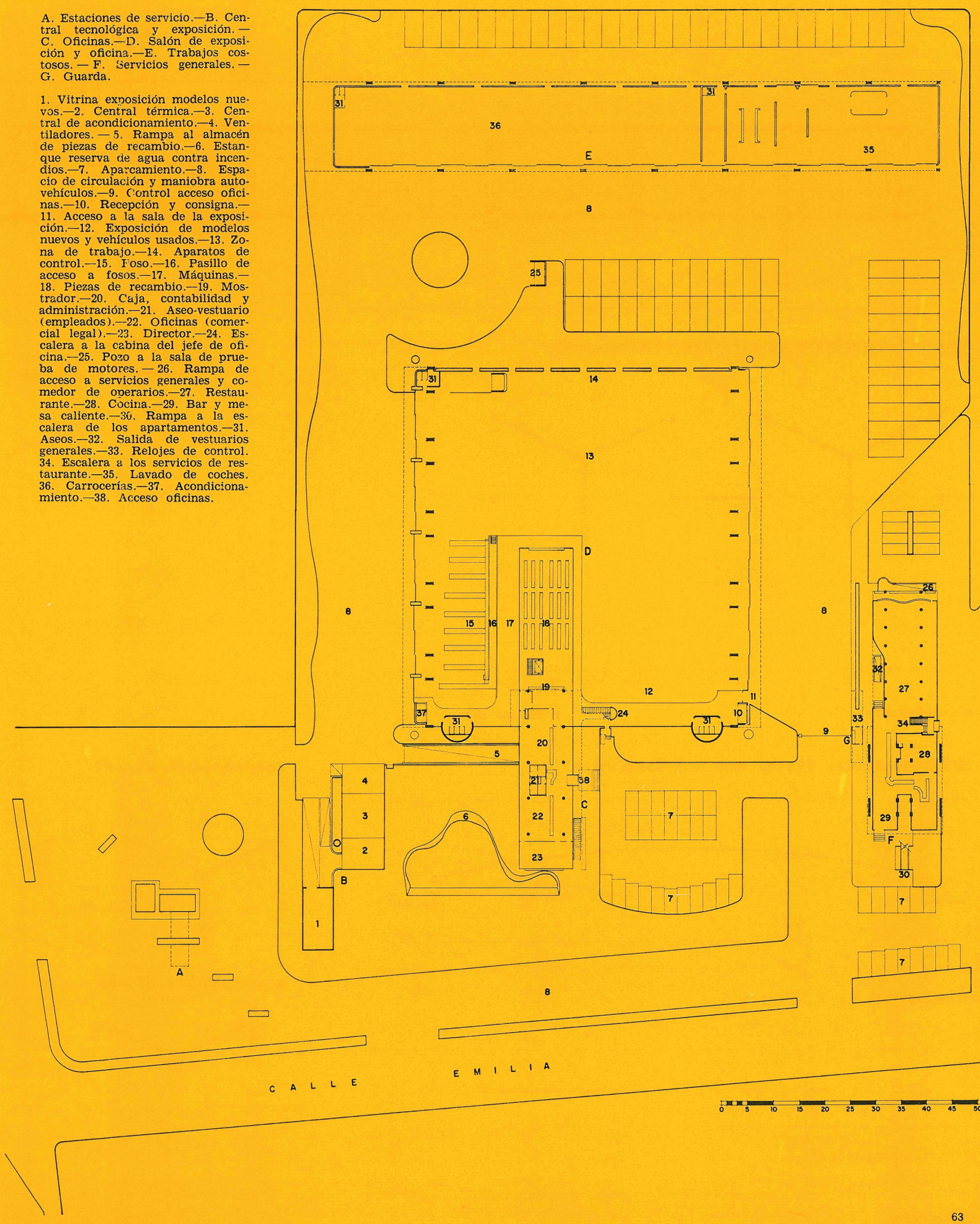

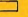

C A L L E

E M I L I A 


\section{seckión longiftudinal}

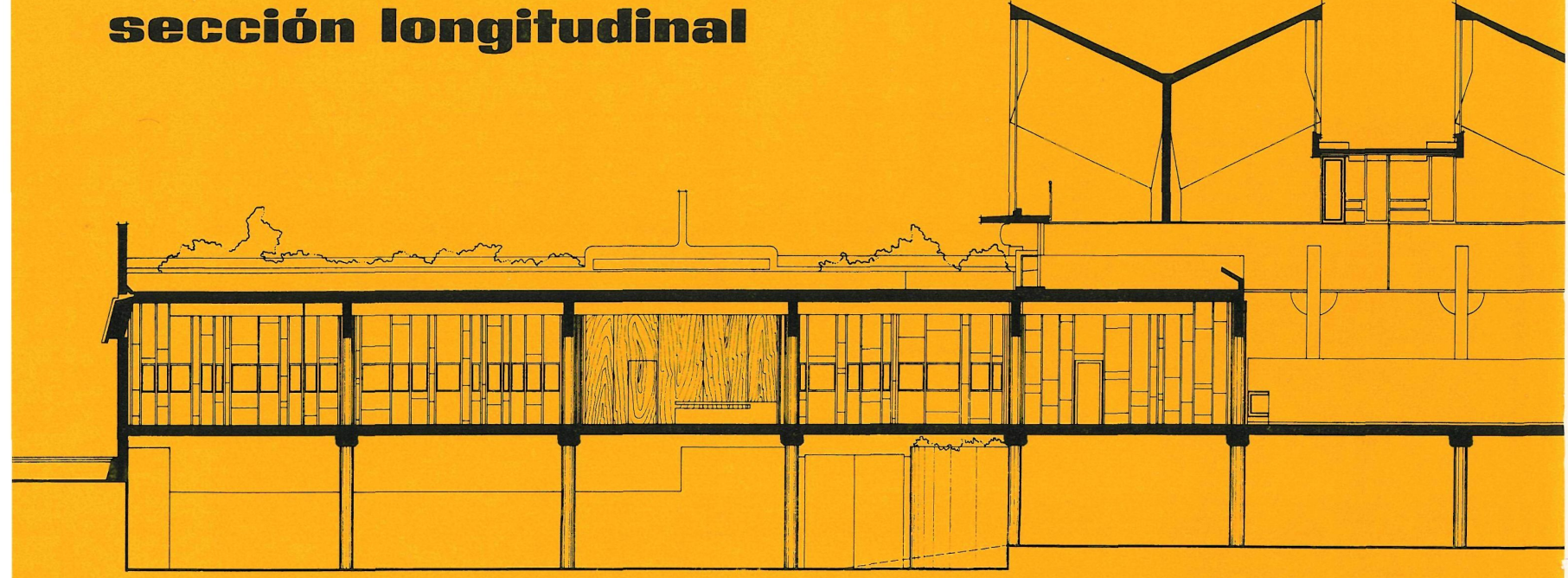

\section{ilumimación y climmatizackión}
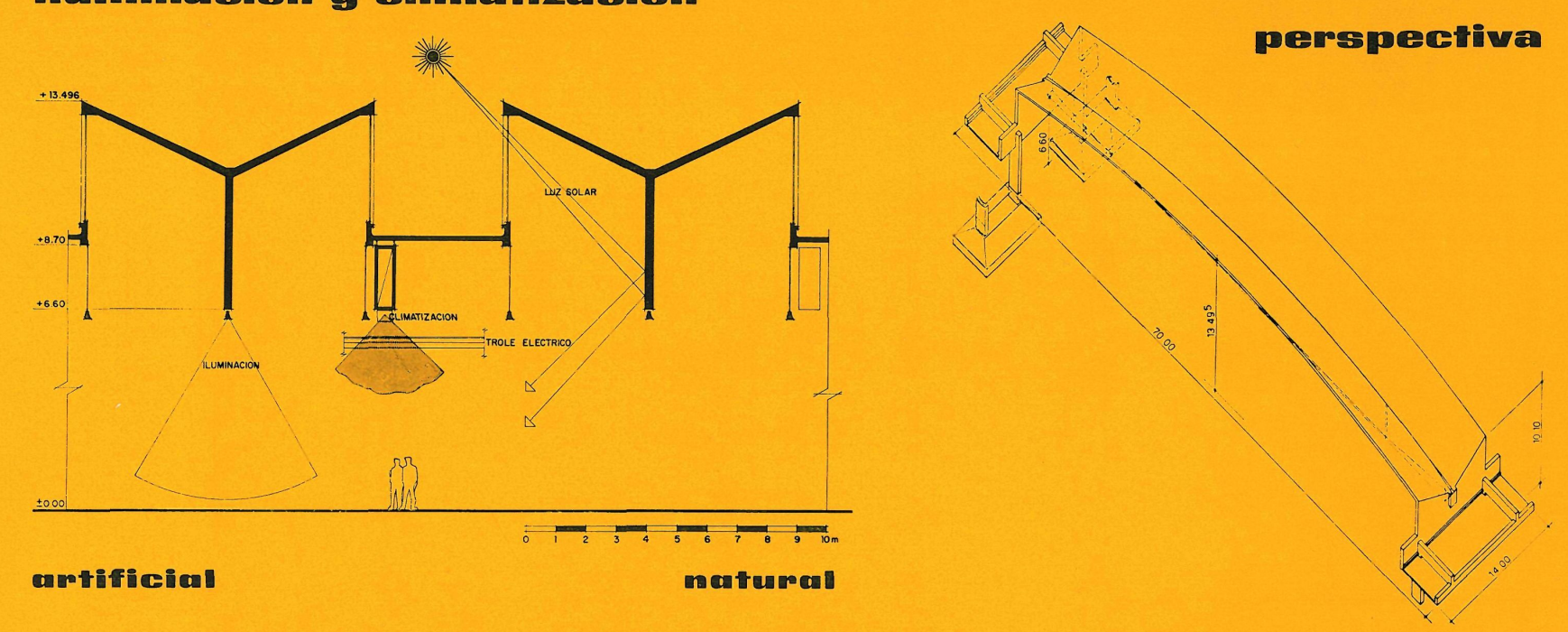

Elemento tipo de cubierta

Sección A - A

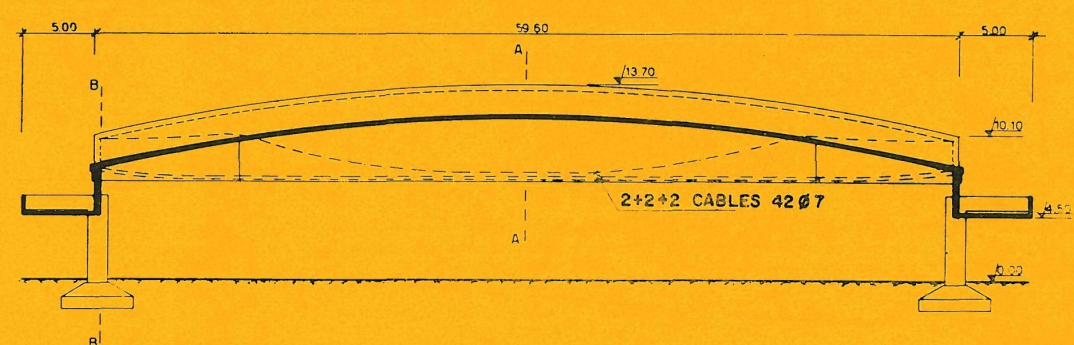

sección

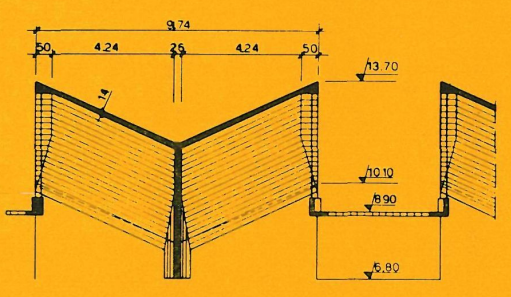

Sección B - B
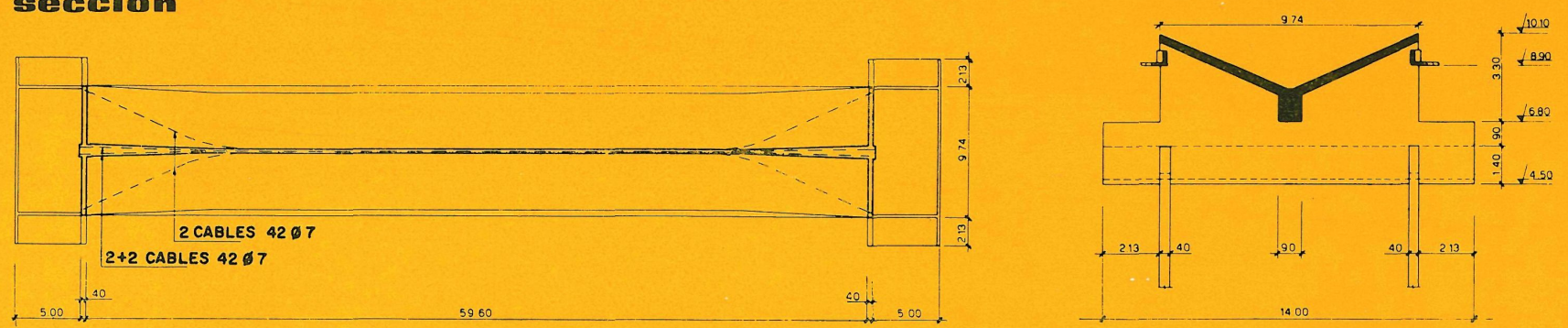

poleanta 


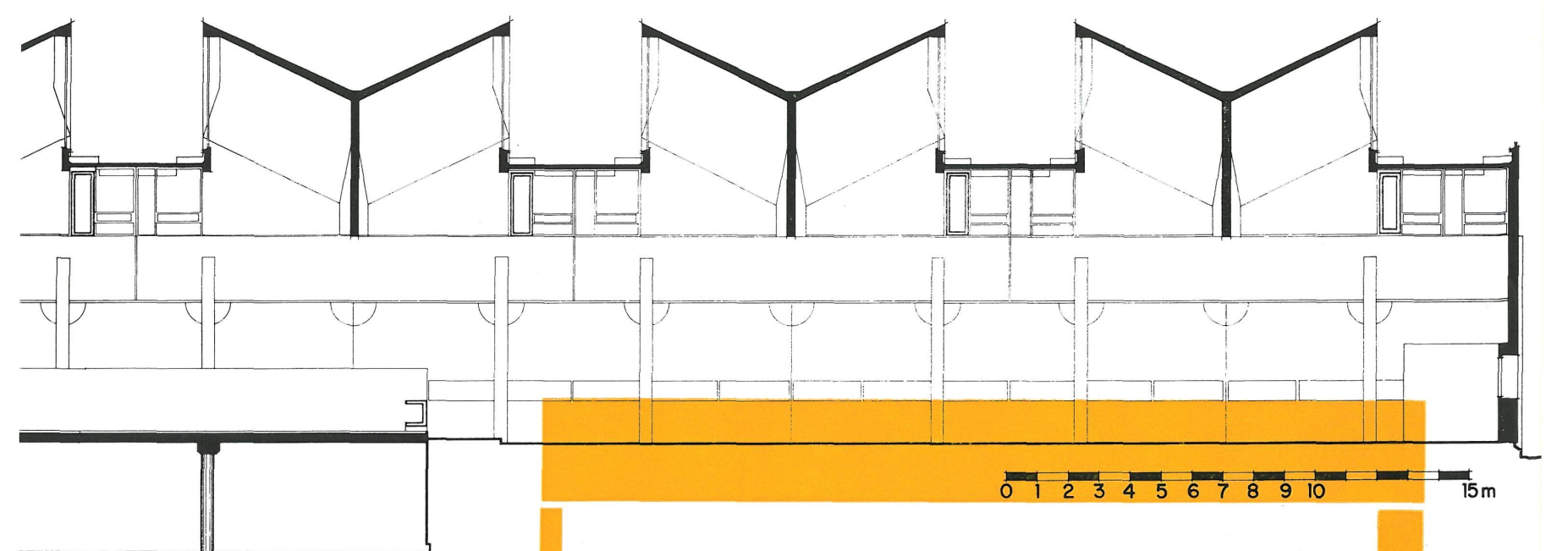

La edificación que contiene los servicios generales y particulares comprende, a su vez, y sin interferencia entre ellas: la zona destinada a los servicios generales de los operarios, ubicados en el semisótano; y la zona ocupada por el bar mostrador para comidas calientes, restaurante con sus servicios adecuados, espacios a los que puede acceder el cliente y disfrutar de una espera cómoda mientras le es entregado su vehículo que estaba en revisión.

El edificio de oficinas aloja: los despachos de la dirección, oficinas comerciales y de representación, además de las oficinas de la sección de administración, departamento de relaciones públicas, la de ventas en el acto, piezas de recambio. Está caracterizado arquitectónicamente por su vigorosa estructura, realizada a base de hormigón «visto».

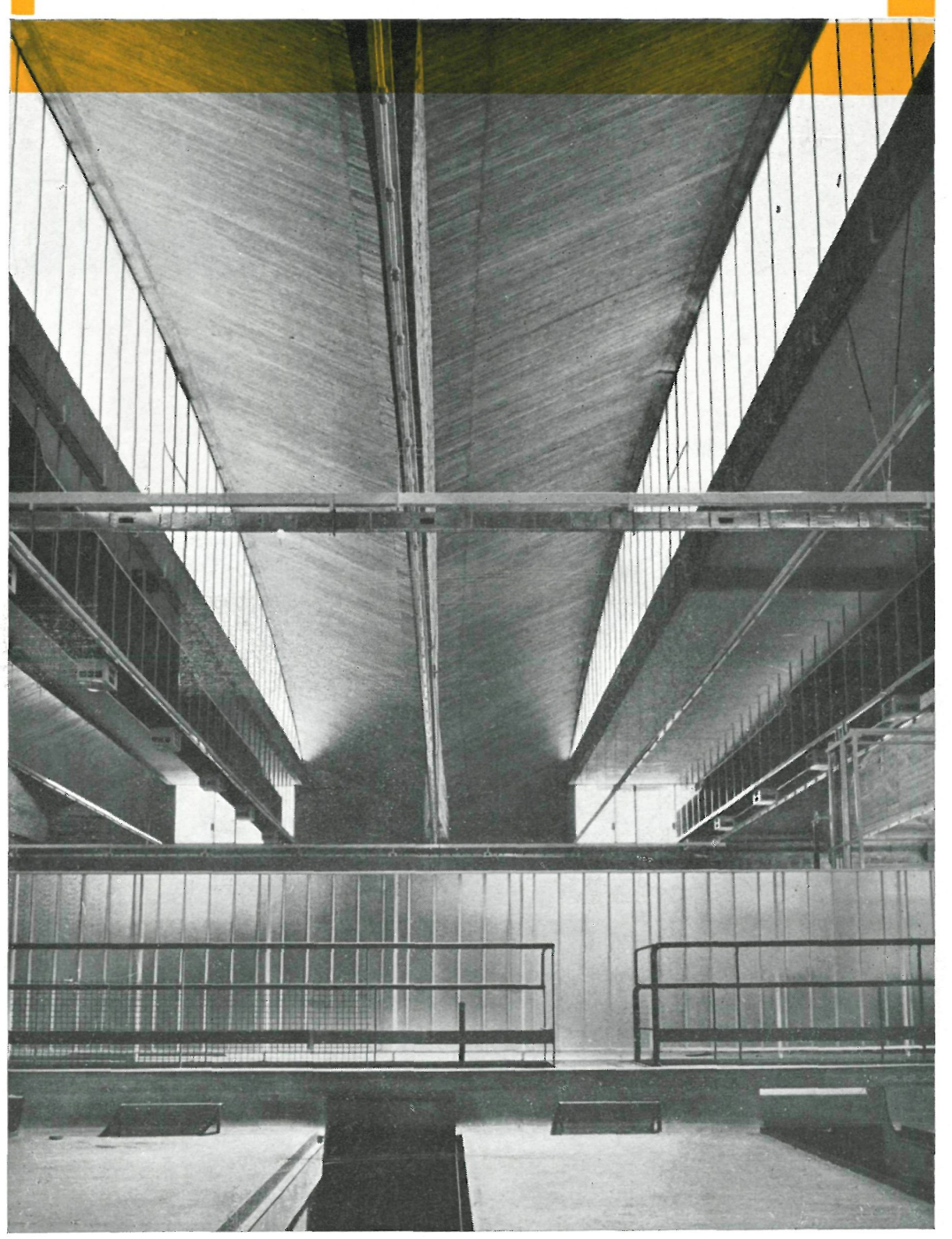




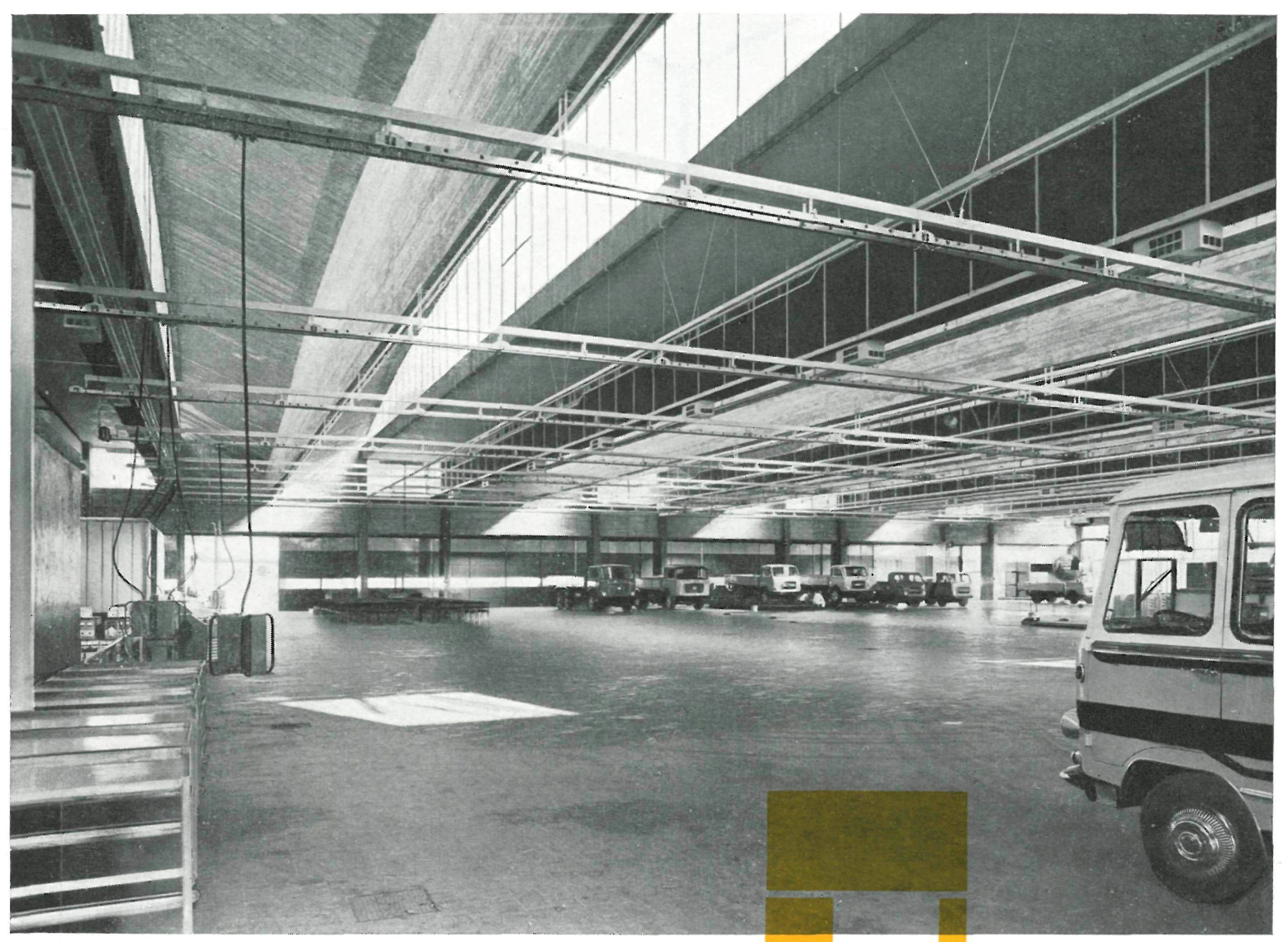

Escalera de acceso a la oficina del jefe de taller.

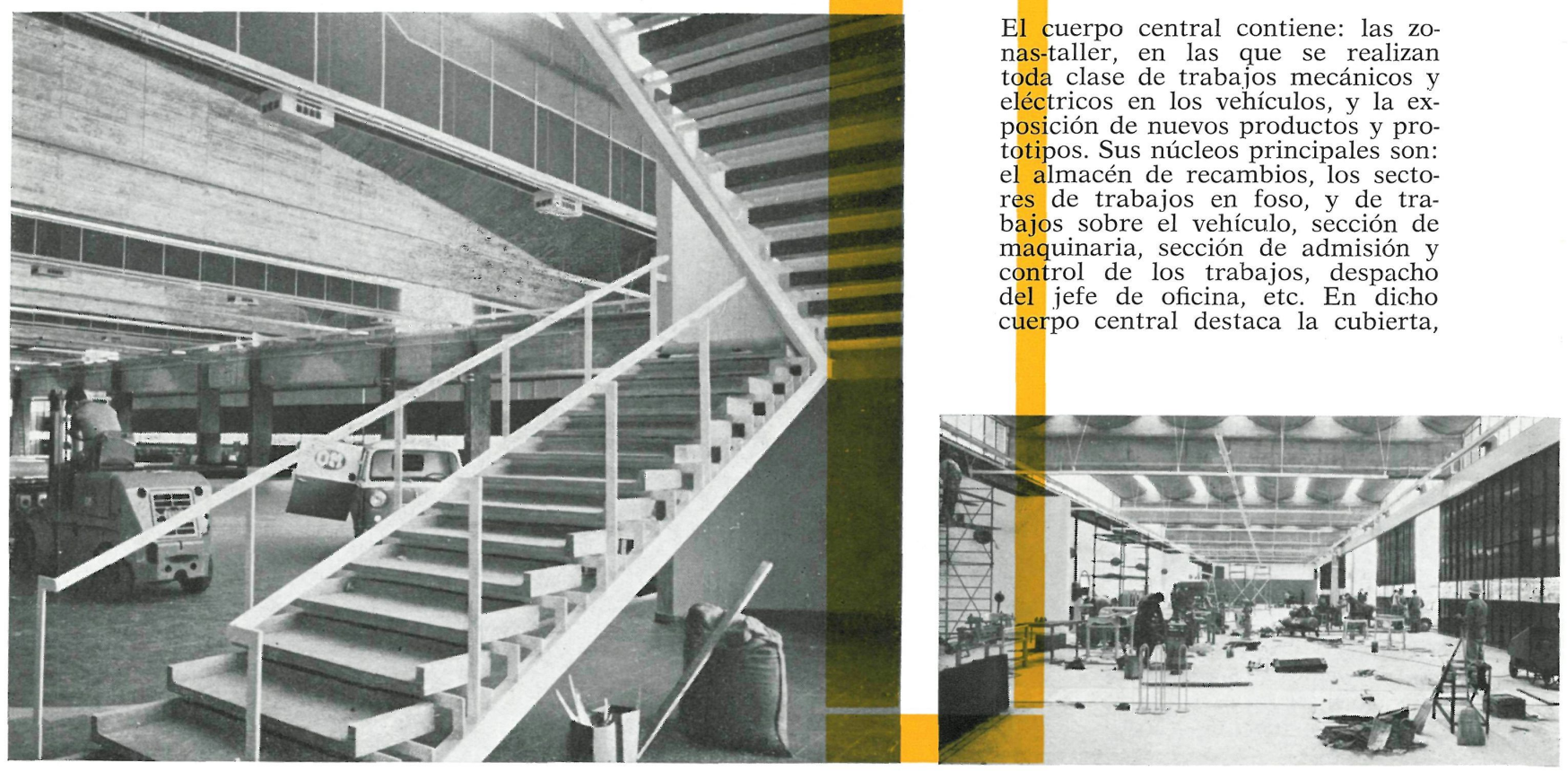




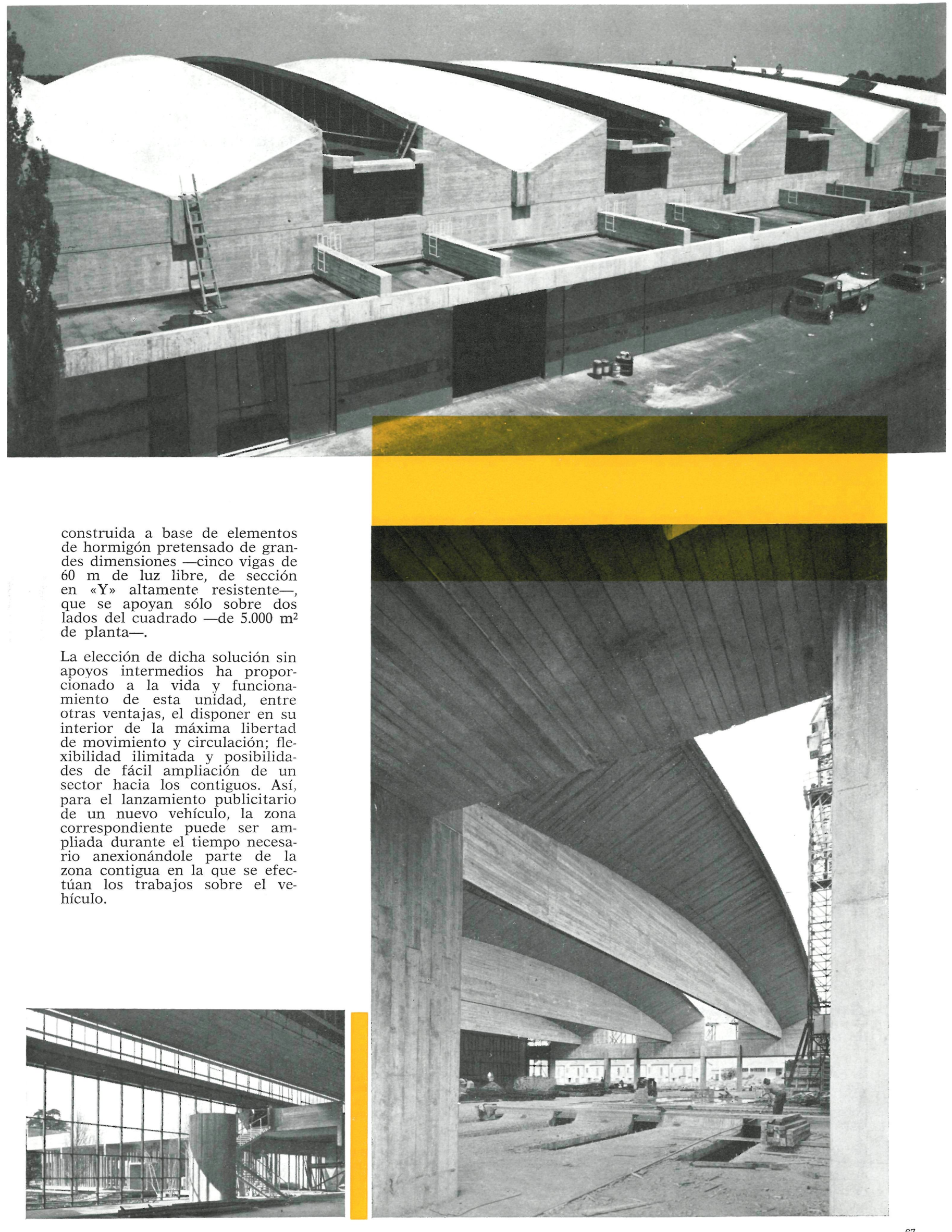


La edificación que alberga la sección de «trabajos pesados» cubre una superficie de cerca de $2.100 \mathrm{~m}^{2}$. En la ejecución de su estructura han sido utilizados elementos standard prefabricados - con forma de hiperboloides-v vigas pretensadas y prefabricadas, y en su interior se efectúan los trabajos específicos de carrocería y lavado, etc. Para imprimir la máxima agilidad a cada zona de trabajo sin obstaculizar las operaciones que se llevan a cabo en los sectores contiguos, toda la fachada, de cerca de $120 \mathrm{~m}$, está dotada de puertas correderas tipo hangar.

E1 edificio de servicios se distingue por un robusto armazón estructural a base de hormigón armado y por una precisa diferenciación de las funciones distintas que allí tienen lugar. En el semisótano se ubican los vestuarios los grupos de duchas, y lavabos, W.C. y el comedor del personal. En la planta baja se distribuyen: el bar, mostrador de comidas calientes, y el restaurante para clientes, disponiendo, además, de los locales y servicios adecuados para que el cliente mientras espera la revisión de su coche pueda disfrutar del confort necesario. La planta superior contiene 10 apartamentos, ocupados por los empleados mayormente ligados a la vida del complejo, que gozan de la adecuada autonomía, libertad y acceso independiente.

La solución adoptada para el conjunto asegura a este Organismo industrial, además de un perfecto racionalismo en su funcionamiento - en línea con los más actuales criterios organizadores-, una alta cualificación; la simplicidad comercial de la administración se compagina con una selecta arquitectura, lo cual ha dado como resultado un éxito técnico-funcional a la vez que un producto de calidad arquitectónica y de personalidad expresiva.

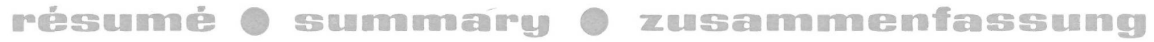

\section{Centre de semvice et d"ussistance pour vélnicules - Itrallie}

G. Gresleri, Dr. architecte

Il s'agit de quatre bâtiments de service complète, le bâtiment de bureaux, reliés à l'entrepôt de pièces de rechange, le bâtiment central occupé par les ateliers où sont effectués les «travaux lourds» -carrosseries, radiateurs, lavage et graissage-, et le bâtiment destiné aux services généraux et particuliers.

Lors de la conception de cet ensemble, ont été étudiés avec attention les aspects suivants: relation client-administration, le travail en tant que facteur éducatif et d'amélioration, et l'homogénéité des qualités aux différents noyaux. La solution architecturale adoptée respire, en plus d'une parfaite rationalité quant à son fonctionnement, une personnalité expressive.

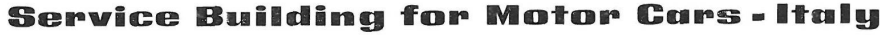

G. Gresleri, Dr. architect

The following units can be separately identified in this building. Complete service station. Office building, and associated to it a spare parts store. The central building, which includes heavy duty workshop, body workshop, radiator department, washing and greasing section, and the department devoted to general and special services.

The following aspects were carefully considered when designing this project. Relation between client and firm. Work as an educational and formative factor. The homogeneity of the various departments. The resulting architectural solution is highly rational, and expresses a powerful personality.

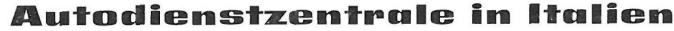

Dr. G. Gresleri, Architekt

In dieser Zentrale können wir folgende Einheiten unterschcheiden: eine komplette Tankstelle; ein mit dem Zubehör lager verbumdenes Bürogebäude; den Baukörper, der den Mittelpunkt des Komplexes darstellt, in dem räumlich abge trennt- die verschiedenen "groben Arbeiten» ausgeführt werden, d.h. Arbeiten an Karrosserien und Kühlern, abschmieren und waschen, usw.; und schliesslich das Gebäude, welches die allgemeinen und individuellen Versorgungsanlagen enthält; usw.

Bei dem Entwurf dieses Komplexes wurden dem Verhältnis Kunde Unternehmung sowie den erzieherischen und anspornenden Aspekten der Arbeit und dem Bestreben nach qualitativ gleichwertiger Bauausführung der verschiedenen Arbeitsplätze besondere Bedeutung beigemessen. Die Verwirklichung dieses architektonischen Gedankens ist nicht nur funktionsmässig rationell sondern auch von origineller Ausdrucksform. 